\title{
Wavelet-based time-delay estimation for time-resolved turbulent flow analysis
}

\author{
M. Jakubowski, ${ }^{\text {a) }}$ R. J. Fonck, C. Fenzi, and G. R. McKee \\ Department of Engineering Physics, University of Wisconsin, Madison, Wisconsin 53706
}

(Presented on 21 June 2000)

\begin{abstract}
A wavelet-transform-based spectral analysis is examined for application to beam emission spectroscopy (BES) data to extract poloidal rotation velocity fluctuations from the density turbulence data. Frequency transfer functions for a wavelet cross-phase extraction method are calculated. Numerical noise is reduced by shifting the data to give an average zero time delay, and the applicable frequency range is extended by numerical oversampling of the measured density fluctuations. This approach offers potential for direct measurements of turbulent transport and detection of zonal flows in tokamak plasma turbulence. (C) 2001 American Institute of Physics.
\end{abstract}

[DOI: 10.1063/1.1326009]

\section{INTRODUCTION}

Turbulence in magnetically confined plasmas continues to be one of the most important and scientifically challenging research topics in controlled thermonuclear fusion research. Turbulence manifests itself as fluctuations in plasma parameters such as the density, temperature, and electrostatic potential. Beam emission spectroscopy (BES) was developed to measure long-wavelength $(k \rho<1)$ density fluctuations in the core and edge regions of tokamak plasmas, and has produced unique observations on the Tokamak Fusion Test Reactor (TFTR), ${ }^{1}$ TEXT $^{2}$ and DIII-D ${ }^{3}$ experiments. BES measures the local density by observing the collisionally excited emission from a deuterium heating neutral beam interacting with plasma electrons and ions. At present, the DIII-D BES system consists of 32 spatial channels, with optics and neutral beam-sightline geometry optimized to allow spatial resolution $\leqslant 1 \mathrm{~cm}$ in the radial and poloidal directions.

In addition to density fluctuations themselves, BES data may be used to extract poloidal rotation velocity fluctuations through time-resolved correlation measurements. This technique derives a time lag between two poloidal channels via a time-resolved cross phase measurement. ${ }^{4}$ The time-varying time lag is directly related to the time-varying poloidal rotation velocity $\tilde{v}_{\theta}(t)$,

$\tilde{\tau}(t)=\Delta z / \tilde{v}_{\theta}(t)$

where $\Delta z$ is the BES spatial channel separation in the poloidal direction. Under the assumption that diamagnetic flows are small compared to rotation speeds, as is typical for large tokamak plasmas $\left(v_{i}^{*}<0.1 v_{E \times B}\right)$, the apparent poloidal speed of the fluctuations in the laboratory frame is given by the radial electric field $\left(E_{r}\right)$ as

$$
\tilde{v}_{\theta}(t)=\tilde{E}_{r}(t) / B_{\phi} .
$$

In general, BES fluctuation spectra are dominated by this rotation-induced Doppler shift, and hence show a linear de-

${ }^{a)}$ Electronic mail: mjakubow@students.wisc.edu pendence between the cross phase and frequency. Hence, $\widetilde{\tau}(t)$ is independent of frequency to first approximation.

The use of a wavelet transform to derive $\tilde{\tau}(t)$ and hence $\tilde{v}_{\theta}(t)$ gives a means of observing rapid variations in plasma flow speeds. At moderate time resolution, this can provide data on the formation of internal transport barriers ${ }^{3}$ and their relation to the local flow velocity shear. If the poloidal rotation velocity fluctuation can be obtained on plasma turbulence-relevant scales (few $\mu$ s), we then obtain the fluctuating radial electric field and indirectly the electrostatic potential on time scales relevant to transport. This may provide direct measurement of the turbulence-driven anomalous cross-field particle transport in tokamaks, given by

$$
\Gamma_{\text {anom }}=\left\langle\tilde{n} \tilde{v}_{r}\right\rangle \propto\langle\tilde{n} \tilde{\Phi}\rangle .
$$

To that end, we examine the ability of wavelet transform cross correlations to extract time variations in the lag time between poloidally adjacent BES sample volumes. Wavelet transform time-delay estimation (TDE) methods are chosen over traditional Fourier transform TDE methods to take advantage of the wavelet capability of measurements with higher time resolution. This article extends preliminary work on simplified sinusoidal time delay oscillations ${ }^{4}$ to more realistic and complete simulations involving turbulent time delays. We conclude that extracting time-delay fluctuations between two time signals is achievable to $\frac{1}{6}$ of the sample frequency. This method may be applied to other analysis problems where fast ( $\geqslant 6$ sample points) time-varying time delay extraction between two correlated signals is required.

\section{SPECTRAL ANALYSIS OF DENSITY AND VELOCITY FIELDS}

Localized BES density fluctuations are typically analyzed via Fourier transform methods to obtain time-averaged power spectra and correlations in the density field. Extending correlation analysis to embody time-resolved phenomena may be achieved by wavelet analysis. ${ }^{5}$

The complex wavelet auto- and cross-power spectra are defined as 


$$
W_{\mathrm{ab}}(f, \tau)=W_{a}^{*}(f, \tau) W_{b}(f, \tau),
$$

with $a=b$ and $a \neq b$, respectively, where $W_{a, b}$ are the individual wavelet transforms, the time-resolved analogs of the Fourier transform in frequency $(f)$ space. Moreover, the timeevolving cross phase (to be used in rotation velocity fluctuations) is given $\mathrm{by}^{5}$

$$
\Theta(f, \tau)=\tan ^{-1} \frac{i m(W(f, \tau))}{r e(W(f, \tau))} .
$$

From a wavelet cross-power spectrum between two adjacent poloidal BES channels, one obtains a time-varying linear phase function of frequency which is proportional to the time delay, ${ }^{1}$

$$
\tilde{\tau}(t)=\Delta \phi_{f}(t) / 2 \pi f .
$$

The linear fit of $\Delta \phi_{f}$ versus frequency is weighted by the amplitude of the density power spectral distribution to reflect the weighting of density carrier contributions to time delay extraction, where the density carrier signal pair contains the time delay relationship information between the two signals. Performing this fit at each point in time yields a timeresolved time delay, and therefore a fluctuating poloidal velocity $\tilde{v}_{\theta}(t)$ from Eq. (1). The essence of the method is accurate extraction of a linear phase function fit in frequency to high time resolution using time-dependent correlation analysis. The temporal resolution of the fit is limited by the time resolution of the wavelet transform.

\section{TURBULENT VELOCITY SIMULATION AND TRANSFER FUNCTION}

To determine the range of frequency in $\tilde{v}$ over which the method is applicable, we use simulated velocity fluctuation data to obtain a point response transfer function and a broadband response function in frequency.

The first step is to simulate a broadband velocity fluctuation properly by imposing a broadband time delay relationship between two signals. Such velocity fluctuations are obtained by starting with a simulated density signal $\tilde{n}_{1}(t)$ defined as the inverse Fourier transform of a broadband Gaussian amplitude distribution in frequency space $\left[A_{\tilde{n}}(\omega)\right]$ with random phase $\theta_{\tilde{n}}(\omega)$,

$$
\tilde{n}_{1}(t)=\int_{-\infty}^{\infty} A_{\tilde{n}}(\omega) e^{i \tilde{n}_{\tilde{n}}(\omega) t} e^{i \omega t} d \omega .
$$

A broadband time delay fluctuation $\tilde{\tau}$ is obtained similarly, but with a distinct $A_{\tilde{v}}(\omega)$ and a statistically independent $\theta_{\tilde{v}}(\omega)$. To generate the second density signal $\tilde{n}_{2}(t)$, correlated with $\widetilde{n}_{1}(t)$ but delayed by $\widetilde{\tau}(t)$, one must impose the desired time-delay fluctuation on the original signal $\tilde{n}_{1}(t)$. This is achieved by applying the appropriate time-delay generator via the sinc function to create a time-delayed version $\tilde{n}_{2}(t)$ from $\tilde{n}_{1}(t):^{6}$

$$
\tilde{n}_{2}(t)=\int_{-\infty}^{\infty} \operatorname{sinc}(\tilde{\tau}(t)+\tau) \tilde{n}_{1}(t-\tau) d \tau
$$

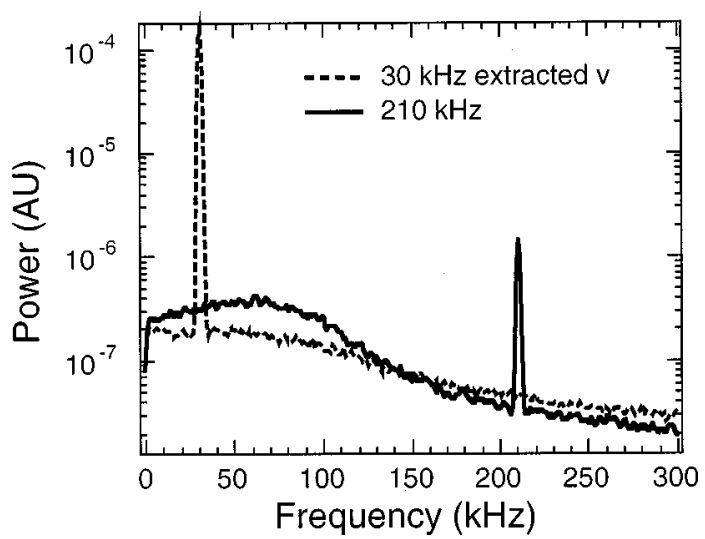

FIG. 1. Response functions for single-frequency time-delay oscillations at 30 and $210 \mathrm{kHz}$ with $f_{\text {sample }}=1 \mathrm{MHz}$.

where $\tilde{\tau}$ is the broadband time-delay fluctuation of interest. This simulates a realistic time delay as expected to be found in the microturbulence measurements in tokamaks.

As an aside, we note that it is critical to properly treat time delays which are longer than one period of oscillation. Given that a linear fit is made to the cross phase between two signals, a difference of more than one period results in a mod- $\pi$ phase jump in frequency space. This is inherent to typical BES data (though this may not be an issue for other experiments), where a time delay on the order of three sample points exists between two adjacent poloidal $\tilde{n}$ channels. A linear fit to a phase difference with $\bmod -\pi$ jumps results in a large increase in the noise in the cross-phase measurement. The physical analogy is that one cannot tell whether a sinusoidal oscillation is leading another oscillation by phase $\phi$ or $\phi$ plus any integer multiple of $2 \pi$.

As an example, the extracted frequency spectrum of a model input flat $0-30 \mathrm{kHz}$ broadband time delay (for a sample frequency $f_{\text {sample }}=1 \mathrm{MHz}$ ) appears as a decaying exponential power spectrum, extending beyond $150 \mathrm{kHz}$. The resulting noise level is at least 200 times greater than the input signal of interest. A decaying exponential is the typical result of a failed time delay reconstruction: it shows none of the inherent spectral characteristics of the input.

To avoid these unphysical jumps in the cross phase, a dc shift of the time delay is imposed on the data to give a mean zero time delay over the period of measurement. The dc shift level is obtained from normal time-delay correlation measurements averaged over the observation time. This shiftcorrection eliminates the unphysical mod- $\pi$ phase jumps and allows recovery of the original input time-delay spectrum.

Given the time delay generator [Eq. (7)] and the dc shift correction, we are able to examine the point response frequency transfer function $T(f)$ for near-sinusoidal time-delay input functions. A sample result is shown in Fig. 1. At 30 $\mathrm{KHz}$ (for $f_{\text {sample }}=1 \mathrm{MHz}$ ), a sinusoidal phase oscillation is recovered cleanly, but the response at $210 \mathrm{kHz}$ is more than 30 times weaker in power. Note that power leakage is manifested as nonzero recovered power in $\widetilde{\tau}(f)$ outside of the imposed frequency range.

Sinusoidal $\tilde{\tau}$ simulations give a frequency transfer function $T(f)$ and leakage power function $L(f)$ normalized to 


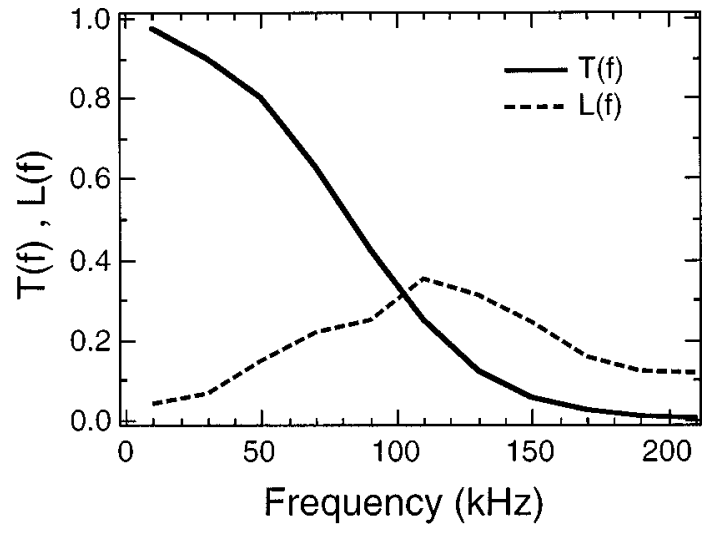

FIG. 2. Transfer and leakage power function for wavelet time-delay extraction from single frequency input functions.

input power (Fig. 2), where leakage power $L(f)$ is defined as the fraction of power measured outside of the input frequency range. A rapid dropoff of response occurs above 50 $\mathrm{kHz}$ or $f / f_{\text {sample }}=0.05$. The frequency response limit is roughly $50 \mathrm{kHz}$ or $f / f_{\text {sample }}=0.05$ if we require $T(f)$ $>75 \%$ and $L(f)<25 \%$.

Given that the estimation of time delay between two turbulence measurements requires a finite sample time to determine the cross phase, it is not surprising that the effective frequency range for the time delay estimation is of the order $f / f_{\text {sample }}=0.05$. However, this is a severe limit on an experiment in that the required sample rate must be increased by an order of magnitude to recover the original frequency range of the background density turbulence (where $\tilde{n}$ measurements are available up to the Nyquist frequency, $f / f_{\text {sample }}=0.5$ ).

This requirement for a substantially increased sample rate may be relaxed by the use of numerical interpolation of the original data to simulate oversampling. The utility of such an approach is evaluated here using the model data generator.

The interpolation of data between successive sample points consistent with a dynamic time delay has been developed by Chan et al., ${ }^{6}$ and here we apply it to provide simulated oversampled density waveforms $\tilde{n}_{1}(t)$ and $\tilde{n}_{2}(t)$. The interpolated signal $s_{i}(t)$ is obtained from a discrete signal $s(n)$ by $^{6}$

$$
s_{i}(t)=\sum_{n=-\infty}^{\infty} \sum_{k=-p}^{p-1} \operatorname{sinc}(\tilde{\tau}+k) s(n-k) \operatorname{sinc}(t-n),
$$

where $p$ is the number of points around the time of interest. Given that a certain number of points (5-20) is required for a wavelet to measure a time delay, interpolating initial signals numerically is the equivalent of estimating data oversampling. No new information is added to the signal $\tilde{n}(t)$, but it is reasonable to expect that successively finer interpolation (equivalent to higher simulated sample frequency) will eventually result in added noise to the resultant extracted $\widetilde{t}(t)$.

An example of the effect of $2 \times$ interpolation [i.e., one interpolated datum between successive measurements in $\tilde{n}_{1}(t)$ and $\left.\tilde{n}_{2}(t)\right]$ on the recovery of higher frequency fluctuations is shown in Fig. 3. The amplitude and signal to

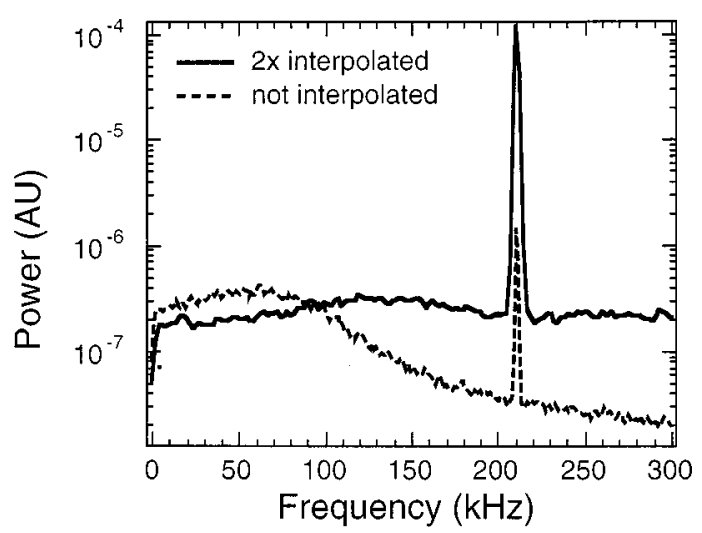

FIG. 3. Effect of twofold interpolation for a sinusoidal time delay at 210 $\mathrm{kHz}$ for $f_{\text {sample }}=1 \mathrm{MHz}$.

background ratio are both recovered for $f \sim 0.2 f_{\text {sample }}$ (cf. Fig. 1).

Transfer functions derived for single frequency oscillations in $\tilde{\tau}(t)$ with successively higher interpolation density are shown in Fig. 4. It can be seen that two- and threefold interpolations increase the sensitivity of the method, while the fourfold interpolation blows up to $T(f) \gg 1$. The two- and threefold interpolations give decaying $T(f)$ which rise slightly higher than unity through a part of the response. The power leakage $L(f)$ out of the desirable reconstruction (the fraction of power in a reconstructed spectrum outside of the peak) is also shown as the upward-sloping curves. One observes that the results improve with up to threefold interpolation, in which case at $f / f_{\text {sample }}=0.15, T(f) \geqslant 75 \%$ and $L(f) \leqslant 25 \%$. Thus we conclude that threefold interpolations are a reasonable limit to increasing the frequency sensitivity, with a frequency range of $f / f_{\text {sample }} \approx 0.15$, or $150 \mathrm{KHz}$ with $f_{\text {sample }}=1 \mathrm{MHz}$.

For the case of $160 \mathrm{kHz}$ white noise without interpolation, the recovered spectrum decays rapidly [Fig. 5(a)], in accordance with the transfer function in Fig. 2, but also shows some sign of power leakage at low frequencies. Oversampling through interpolation recovers the original input

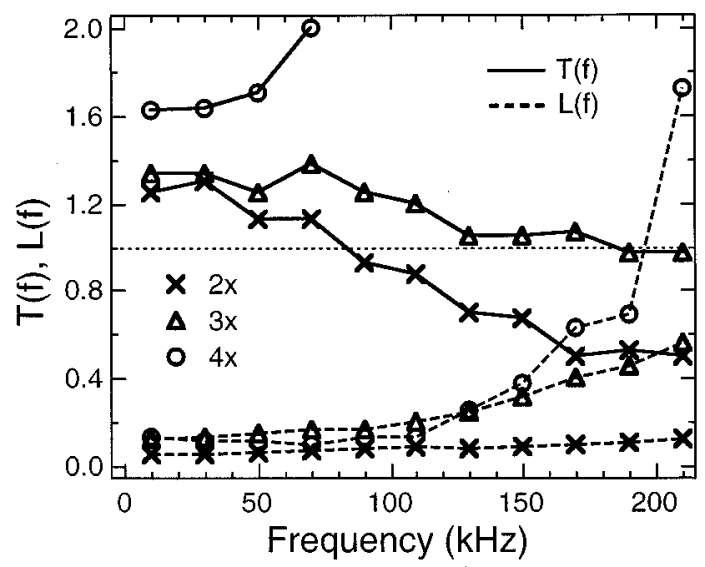

FIG. 4. Transfer functions $T(f)$ and leakage power functions $L(f)$ derived for single-frequency oscillations in $\widetilde{\tau}(t)$ with two-, three-, and fourfold interpolation density. 


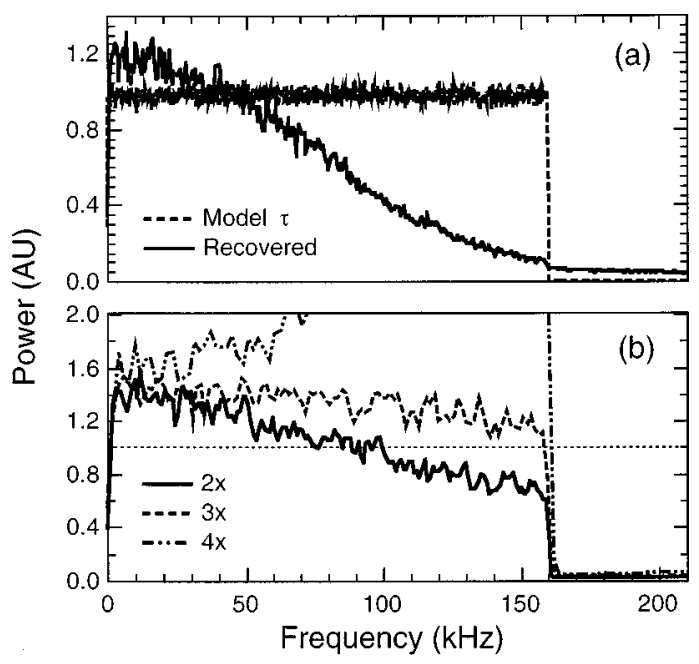

FIG. 5. (a) Model and recovered $160 \mathrm{kHz}$ white input $\widetilde{\tau}(t)$ spectrum. (b) Reconstruction of the white spectrum with two-, three-, and fourfold interpolation density.

broadband spectrum more accurately [Fig. 5(b)], with $3 \times$ interpolation giving a nearly flat spectrum and an average power of $\sim 1.3$ relative to the input. The $4 \times$ interpolation gives a rapidly rising output spectrum, suggesting an increasing injection of excess power at higher frequency.

\section{COMPARISON OF WAVELET AND TIME-DOMAIN TIME-DELAY ESTIMATION METHODS}

Traditional time-domain time delay estimation (TDE) methods require analysis intervals at least as long as the slowest data oscillation, while wavelet methods are able to sample over fractions of an oscillation. By taking advantage of the nature of the wavelet transform, wavelet-based TDE is capable of temporal resolution at least a factor of 10 faster than traditional methods.

Time-domain TDE procedures are based on maximizing the overlap between two signals. A sinc function can be used to model a continuous time delay in one of the discrete signals [Eq. (7)], after which a time delay parameter which gives maximum overlap is extracted at each point in time. Standard TDE theory requires data analysis windows at least one $\tilde{n}$ period in duration because the analysis signal must have zero mean. Therefore, the maximum extractable frequency cannot be larger than the frequency of the slowest $\tilde{n}$ oscillation. This occurs because a fixed-length analysis window provides no adaptation to the $\tilde{n}$ period. Furthermore, for short analysis windows of $\sim 1$ period, this is difficult to accomplish in the presence of noise.

Wavelet analysis lends itself to extracting time delay oscillations at subperiod temporal resolution. One must recall that the time delay is independent of frequency. As such, a wavelet $\psi\left(f_{1}\right)$ may extract a time delay oscillation $\tilde{\tau}\left(f_{2}\right)$, where $f_{1}>f_{2}$. This relies on the fact that a fast density oscillation $\tilde{n}\left(f_{1}\right)$ may carry a slow $\tilde{\tau}\left(f_{2}\right)$ where $f_{1}>f_{2}$, and it is the $\tilde{n}\left(f_{1}\right)$ which the wavelet $\psi\left(f_{1}\right)$ analyzes. In this sense, $\tilde{n}\left(f_{1}\right)$ is independent of $\tilde{\tau}\left(f_{2}\right)$, minimizing the requirement of an analysis window at least one $\tilde{n}$ period in duration.
The wavelet method can be viewed as a correlation with appropriate frequency filtering and windowing. The only difference is that $\tilde{\tau}$ is obtained from phase difference between two signals, not from the peak of the correlation function. The wavelet method automatically gives continuous $\tilde{\tau}$ from a discrete signal, while traditional correlations require the use of error-prone interpolations to obtain subsample $\tilde{\tau}$ resolution.

Numerical interpolation helps to expand the $\tilde{\tau}$ extraction frequency limits in the wavelet method because ultimately the number of sample points determines the speed of the extraction. This is not so in time domain TDE: one still needs a full $\tilde{n}$ oscillation period to determine the time delay, no matter how many sample points exist in that oscillation. As a result, tenfold improvement in frequency resolution is typically obtained with the wavelet method compared to standard TDE.

\section{SUMMARY}

With the combination of dc shift correction and data interpolation, the wavelet phase extraction technique can extract time-delay oscillations $\geqslant 6$ sample points long, or $\leqslant 150$ $\mathrm{KHz}$ at a $1 \mathrm{MHz}$ sampling rate on the present BES system on DIII-D. This method may be applied to any time-varying time-delay analysis problem.

While these initial tests appear promising, two critical issues remain to be evaluated before this approach can be applied to measurements. The first issue is the influence of the nature of the $\tilde{n}$ carrier spectrum on the resulting transfer function of the $\tilde{v}$ extraction. The second is the influence of broadband white noise (arising from amplifier of photon statistics) in the carrier $\tilde{n}(t)$ signals on the resultant $\widetilde{v}$ extraction. Both of these potential complications are presently under study and will be discussed in future reports.

On the experimental side, future plans involve another threefold increase in digitization rate in addition to the threefold numerical oversampling equivalent of a faster digitization rate described in this article. That would bring velocity field measurements up to the frequency response of the present density field in DIII-D BES turbulence measurements, allowing exploration of turbulence-driven transport and possibly direct observation of zonal flow signatures. ${ }^{7}$

\section{ACKNOWLEDGMENTS}

The authors would like to thank Dr. H. C. So of the City University of Hong Kong for valuable discussion on standard time delay estimation methods. This work was supported by U.S. Department of Energy Grant No. DE-FG0289ER53296.

${ }^{1}$ R. J. Fonck et al., Phys. Rev. Lett. 70, 3736 (1993).

${ }^{2}$ R. D. Durst et al., Rev. Sci. Instrum. 66, 842 (1995).

${ }^{3}$ G. McKee et al., Phys. Rev. Lett. 84, 1922 (2000).

${ }^{4}$ M. Jakubowski et al., Rev. Sci. Instrum. 70, 874 (1999).

${ }^{5}$ M. Onorato et al., Eur. J. Mech. B/Fluids 16, 575 (1997).

${ }^{6}$ Y. T. Chan et al., IEEE Trans. Acoust., Speech, Signal Process. ASSP-29, 577 (1981).

${ }^{7}$ K. H. Burrell et al., Plasma Phys. Controlled Fusion 42, A205 (2000). 